\title{
Carbon nanotube sponges as tunable materials for electromagnetic applications
}

\author{
M V Shuba ${ }^{1,2} \ddagger$, D I Yuko ${ }^{1}$, P P Kuzhir ${ }^{1,2}$, S A Maksimenko ${ }^{1,2}$, \\ M De Crescenzi ${ }^{3}$ and M Scarselli ${ }^{3}$ \\ ${ }^{1}$ Institute for Nuclear Problems, Belarus State University, Bobruiskaya 11, 220050 \\ Minsk, Belarus \\ 2 Tomsk State University, Lenin Avenue 36, 634050, Tomsk, Russia \\ 3 Department of Physics, University of Rome Tor Vergata, 00133, Rome, Italy \\ E-mail: mikhail.shuba@gmail.com
}

\begin{abstract}
The microwave conductivity and permittivity of both single-walled and multi-walled carbon nanotube (SWCNT and MWCNT) sponges were measured while compressing the samples. Compression leads to a huge variation of the absorptance, reflectance, and transmittance of the samples. The dependence of the microwave conductivity on the sponge density follows a power-law relation with exponents $1.7 \pm 0.1$ and 2.0 \pm 0.2 for MWCNT and SWCNT sponges, respectively. These exponents can be decreased slightly by the addition of a nonconducting component which partly electrically separates adjacent tubes within the samples. The conductivity of MWCNT sponge was measured in the terahertz range while heating in air from $300 \mathrm{~K}$ to $513 \mathrm{~K}$ and it increased due to an increase of a number of conducting channels in MWCNTs.
\end{abstract}

Keywords: carbon nanotubes, 3D network, microwave absorption, conductivity 


\section{Introduction}

Carbon nanotubes (CNT) are highly conductive and very elongated nanostructures effectively interacting with electromagnetic (EM) radiation $[1,2,3,4,5,6,7]$. Many physical effects have been predicted and demonstrated in CNTs, including: slowed-down surface wave propagation [1], localized plasmon resonance in the terahertz range $[8,5]$, strong screening effect in microwave and radio-frequency ranges [9, 6], and near-field enhancement effect $[6,10]$. CNTs have a potential as elements for different integrated circuits and electromagnetic devices, such as interconnects [11, 12], transmission lines $[1,2]$, antennas $[13,4,14]$, and terahertz emitters and detectors [15]. Moreover, CNTs are actively used as inclusions for composite materials demonstrating excellent electromagnetic performances [16, 10, 17].

The static electrical conductivity of the composite materials versus the tube concentration has been investigated in many papers (see [18] and references therein). These works focused on finding the percolation threshold for different fabrication methods, dielectric matrices and types of inclusions. To study the influence of the CNT concentration on the conductivity, one needs to prepare many samples with different CNT volume fractions, varying from $0.1 \%$ up to $10 \%$. Due to an unavoidable agglomeration effect, it is difficult to achieve homogenous distribution of the CNTs at high CNT volume fractions and to reach the same extent of tube dispersion within the composite for small and large tube density. In this regard, we have demonstrated that hybrid films constituted by thick $\mathrm{WS}_{2}$ nanotubes and thin CNTs can have homogenous dispersion of CNTs with volume fraction of up to 5\% [19]. Interestingly it has been shown that the conductivity of these films follows a power laws for static, microwave and terahertz regimes [19].

The microwave response of composites in a wide range of CNT fraction (110 , or 2-25 wt\%) above percolation threshold has been reported in many papers $[20,21,22,23,24,25,19]$. It has been shown that loss tangent of composite materials increases with CNT concentration, but it still remains less than unity [20, 23]. However, the concentration dependence of the microwave conductivity has not been discussed yet. It has been only noticed that, from static regime to microwave range, the conductivity of the composite practically does not depend on frequency at high CNT loading [26, 27], though it is not always true as reported in literature [28, 29].

In this paper we present the microwave study of both single-walled and multiwalled CNT (SWCNT and MWCNT) sponges at different tube densities. CNT sponges are emerging extremely lightweight nanomaterials constituted by random and selfsupporting 3D networks. The sponge density can be increased by 20 times starting from $5-20 \mathrm{mg} \mathrm{cm}^{-3}$ by means of a compression force applied along selected direction. In this way, it is possible to obtain the density dependence of the microwave conductivity for one sample only. We found a power-law dependence of the conductivity on the tube density in agreement with the 3D network theory above percolation threshold [30].

Note, that static electrical resistivity of the sponges changes linearly and reversibly 
after 300 cycles of large-strain compression suggesting potential applications as strain sensors and conductive nanocomposites [31]. The use of CNT sponges has been proposed also for pressure-sensing [32], in photon-energy conversion devices [33], for oil absorption [34], for the removal of toxic organic solvents (e.g. o-dichlorobenzene) from water [35].

The main result in this paper is that the CNT sponge compression leads to a decrease of the absorptance and a sizable increase of the reflectance by a factor of four.

\section{Experimental details}

MWCNT sponges were obtained by a chemical vapour deposition (CVD) process. Details of their synthesis and structural and electronic characterization can be found elsewhere [32, 35, 33].

SWCNT sponges were assembled from non-purified bundled single-walled CNTs (OCSiAl Inc.) produced by a chemical vapor deposition process with diameters of $1.5-$ $2.1 \mathrm{~nm}$, and a purity exceeding $75 \%$.

Raman spectroscopy were performed using a Raman spectrometer combined with a confocal microscope Nanofinder High End (Tokyo Instruments) at an excitation wavelength of $532 \mathrm{~nm}$.

Scheme of the microwave measurements. The microwave measurements of the reflectance and transmittance in the range $27-36 \mathrm{GHz}$ were performed by waveguide method using a scalar network analyzer R2-408R (ELMIKA, Vilnius, Lithuania) [36, 37]. The sample was placed in the sample holder of a rectangular $3.4 \times 7.2 \mathrm{~mm}$ waveguide in a plane orthogonal to the direction of $\mathrm{TE}_{10}$ mode propagation. Sponge occupied the whole space between waveguide walls and two parallel $0.1 \mathrm{~mm}$ thin mica plates standing perpendicular to the waveguide axis. Mica plates are completely transparent in the microwave range. Initially, the distance $d$ between mica plates was $3.2 \mathrm{~mm}$, as shown in figure 1. It was reduced yielding a compression of the CNT network with a consequent increase of its density. Microwave measurements have been done for different distances between mica plates in the range from 3.2 to $0.2 \mathrm{~mm}$. The complex relative permittivity $\varepsilon$ and conductivity $\sigma=2 i \pi f \varepsilon_{0} \varepsilon$ of the samples were found using Fresnel formula for a homogeneous slab. Here $f$ is a frequency and $\varepsilon_{0}=8.85 \times 10^{12} \mathrm{~F} \mathrm{~m}^{-1}$.

For terahertz measurements, $0.2 \mathrm{~mm}$ thick film was cut off from uncompressed MWCNT sponge. The complex transmission of this film was measured under normal incidence in the range $0.2-1.2 \mathrm{THz}$ using time-domain terahertz spectrometer (EKSPLA, Vilnius Lithunia). The measurements at $300 \mathrm{~K}, 323 \mathrm{~K}$ and $513 \mathrm{~K}$ were done in air using a home-made furnace for heating sample-holder. The permittivity and conductivity of the samples were calculated by means of the Fourier transform of the measured timedomain signal and application of the Fresnel formula for dielectric slab [41]. 


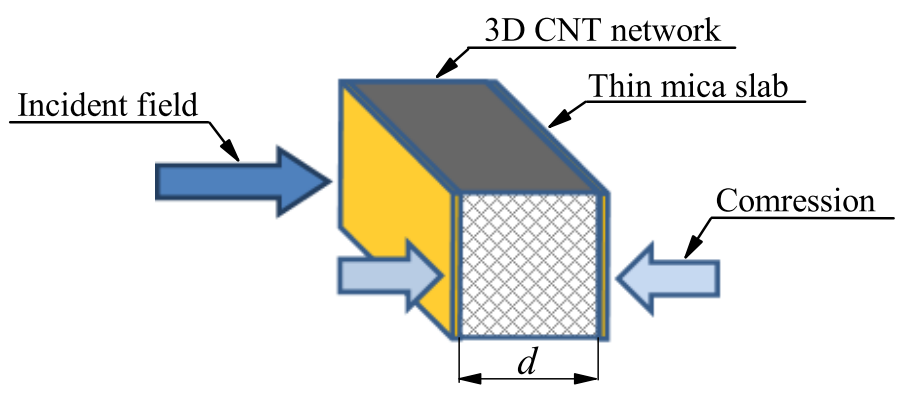

Figure 1. (a) Scheme of the microwave measurements of a 3D CNT network under compression.

\section{Results and discussion}

MWCNT sponges contained a mixture of entangled hollow carbon fibers and MWCNTs (see figure 2(a,b)). Carbon fibers are mainly straight with an outer diameter $0.5-2 \mu \mathrm{m}$ and length up to $0.5 \mathrm{~mm}$, whereas MWCNTs are curved having outer diameter 15-200 $\mathrm{nm}$ and length of tenths of micrometers. Typical sponge density is $35 \mathrm{mg} \mathrm{cm}^{-3}$.

SWCNTs appeared in the form of flakes of 1-5 $\mathrm{mm}$ in size (see figure 2(c,d)). SWCNTs were homogeneously distributed within each flake (see figure 2(e)), and flakes were homogeneously distributed within the SWCNT sponge. We varied the number of the flakes per unit volume and obtained the CNT sponges of different average densities, typically, less than $7 \mathrm{mg} \mathrm{cm}{ }^{-3}$. Since SWCNT flakes are not bound with each other, the SWCNT sponge cannot be considered as self-supporting. The density of SWCNTs in the sponge can be varied by addition of a non-conducting component - a cotton wool (see figure 2(f)).

Raman spectra for MWCNTs and SWCNTs are shown in figure 3. The dominant features are a high-frequency G-band (1570-1600 $\left.\mathrm{cm}^{-1}\right)$ and D-band $\left(1320-1350 \mathrm{~cm}^{-1}\right)$. G-band originates from tangential vibrations of $\mathrm{sp}_{2}$-hybridized carbon atoms and Dband is associated with disorder-induced symmetry-lowering effects. Very small ratio of the intensity of G-mode to that of the D-mode $I_{D} / I_{G}=0.02$ indicates high crystalline quality of SWCNTs. On the contrary, high value of $I_{D} / I_{G}=0.9$ for MWCNTs reveals their highly defective crystalline structure.

Figure 4 shows how the reflectance $(R)$, transmittance $(T)$, and absorptance $(A=1-R-T)$ of the plate-form CNT sponges modifies with increasing sponge density $\rho$ as a function of the compression. The transmission of the sample is quite low in the whole range $(27-36 \mathrm{GHz})$, and no interference effect occurs within the sample. This means that the obtained values $A$ and $R$ are very close to those for infinitely thick samples at the same tube density. The value of $\rho$ varies in the range $30-630 \mathrm{mg} \mathrm{cm}-3$ and $10-160 \mathrm{mg} \mathrm{cm}^{-3}$ for MWCNT and SWCNT sponges, respectively.

One can see from figure 4 , that the variation of parameters $A, R$, and $T$ with density is qualitatively similar for both types of the samples. The reflection increases 

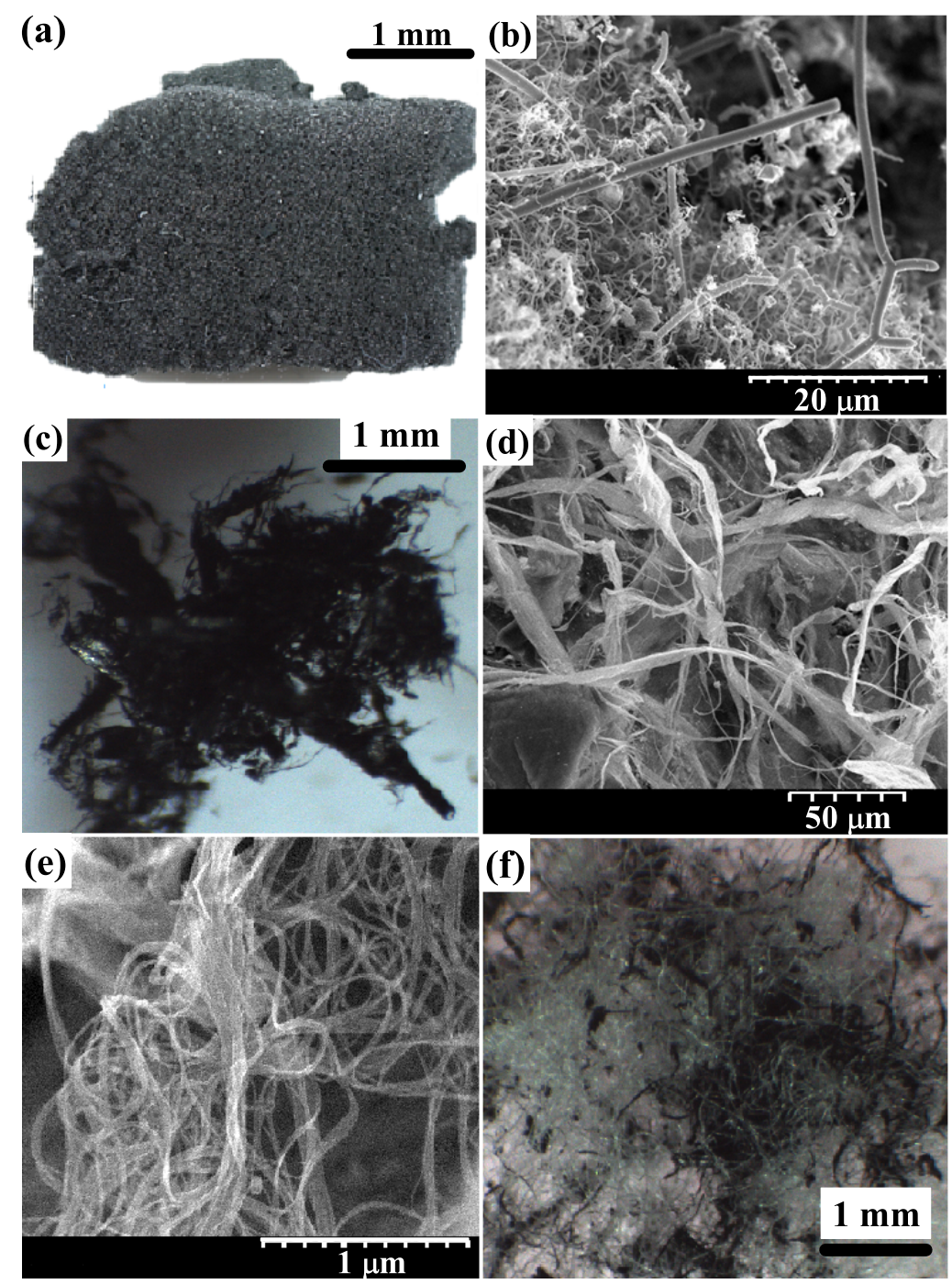

Figure 2. Optical and SEM images of (a,b) MWCNT sponge and (c,d,e) SWCNT flake. (f) Optical image of a mixture of black SWCNT flakes and white cotton wool.

with density in a wide range from $22 \%$ up to $74 \%$ and $94 \%$ for MWCNT and SWCNT sponges, respectively. Accordingly we measured strong changes in absorptance, in fact it decreases from $78 \%$ down to $26 \%$ for MWCNT and from $78 \%$ down to $6 \%$ for SWCNT sponges. Thus, the microwave response of CNT sponges can be tuned continuously by means of an external compression which changes the density of the samples and therefore dramatically varies reflectance and absorptance responses.

Figure 5 shows the density dependence of the permittivity $\varepsilon$ and conductivity $\sigma$ for both types of sponges. The real part of the permittivity is close to unity at low density and becomes negative with increasing density. The compression makes the sponge permittivity to be close to that characteristic for metals. The values $\operatorname{Re}(\sigma)$ (as well as $\operatorname{Im}(\varepsilon)$ ) follow a power-law relation: $\operatorname{Re}(\sigma) \propto \rho^{t}$ with exponent $t=1.78$ and $t=1.95$ for MWCNT and SWCNT sponges, as a function of the density (see figure 


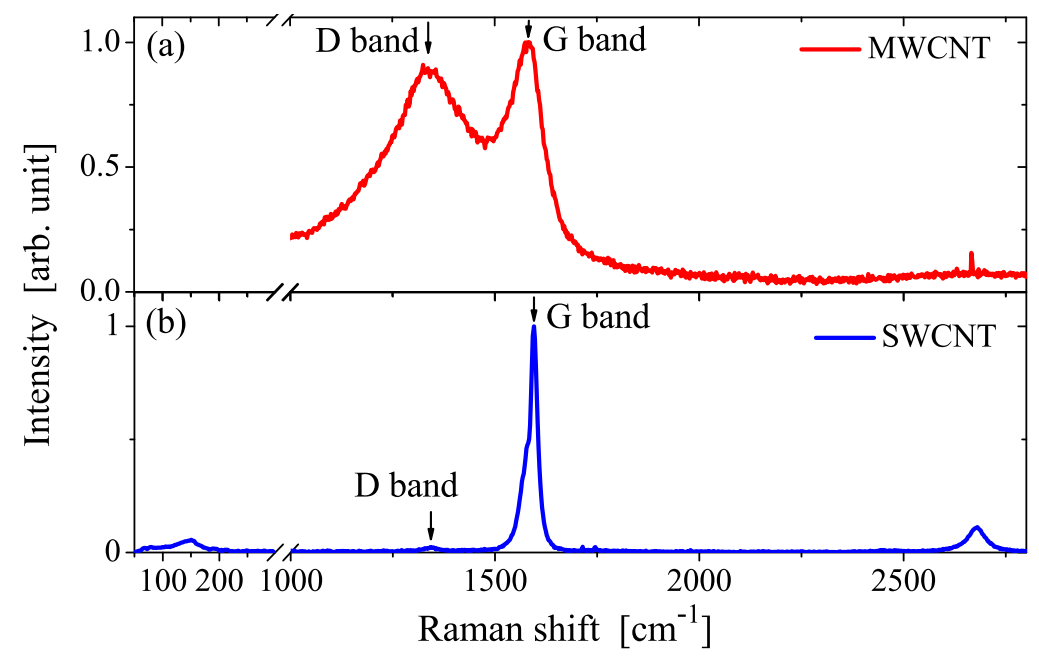

Figure 3. Raman spectra of (a) MWCNT and (b) SWCNT sponges.

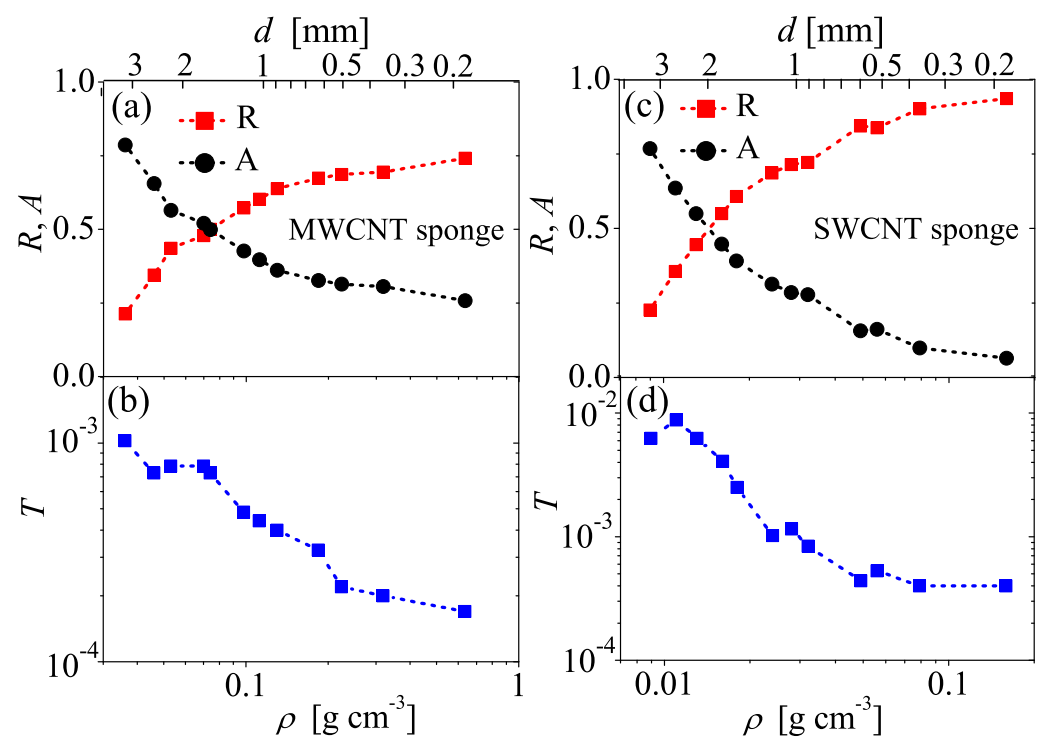

Figure 4. Absorptance (A), reflectance (R), and transmittance $(\mathrm{T})$ versus density $\rho$ and corresponding thickness $d$ of the 3D network of $(\mathrm{a}, \mathrm{b})$ MWCNTs and (c,d) SWCNTs at $32 \mathrm{GHz}$.

$5(\mathrm{c}, \mathrm{f}))$.

Parameters shown in figures 4 and 5 were obtained for one sample with an error $<15 \%$. Since the sponge was irreversibly compressed, we could not do any more measurements of the same sample. Therefore we studied 5 samples of each kind of sponge and found the value of $t$ being in the intervals 1.6-1.8 and 1.8-2.2 for MWCNT and SWCNT samples, respectively. It is noteworthy, that $t$ depends on the frequency. It is maximal in static regime $(f=0 \mathrm{~Hz})$ and decreases with frequency reaching unity in the terahertz range [19]. The value of $t$ for $\mathrm{CNT} /$ polymer composites in static regime is predominantly in the range $1.3-4.0$ peaking around $t=2[18]$. 


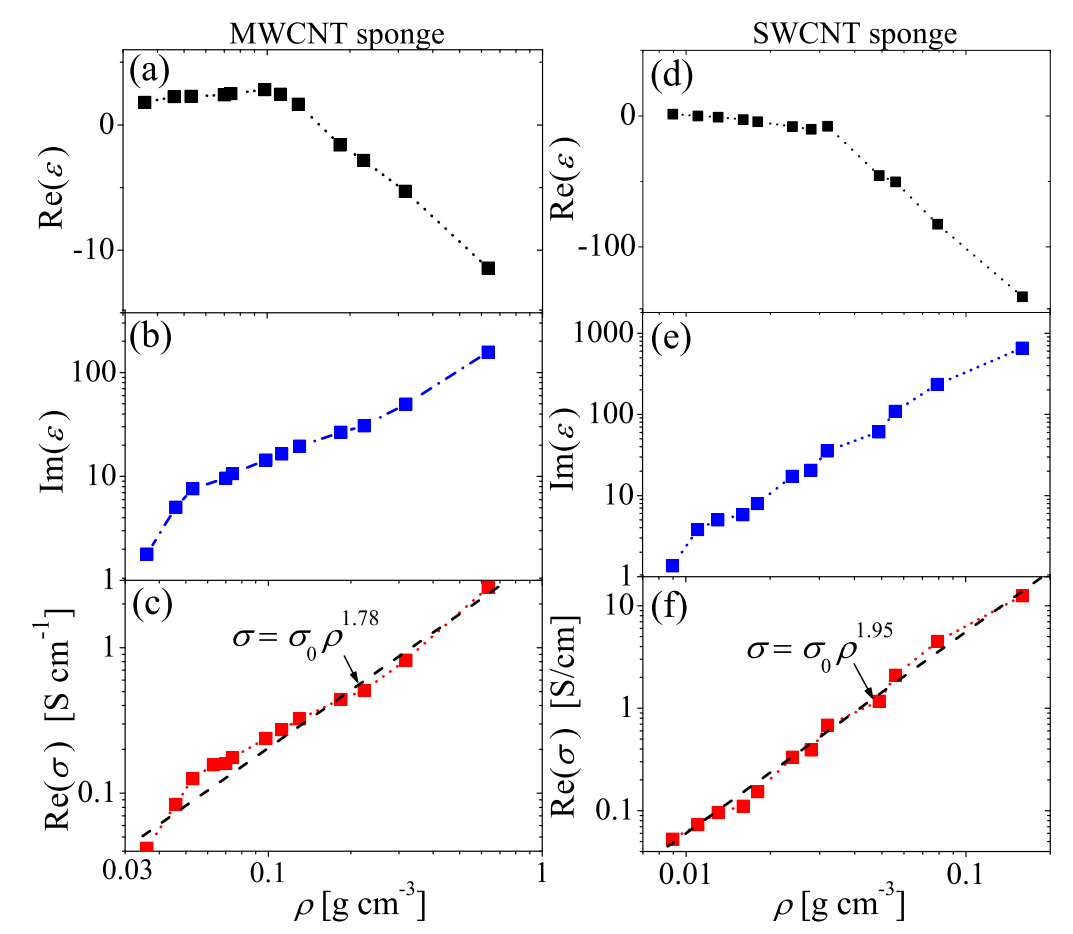

Figure 5. The real and imaginary parts of the permittivity $\varepsilon$ and also the real part of the conductivity $\sigma$ at $32 \mathrm{GHz}$ versus the density of (a,b,c) MWCNT and (d,e,f) SWCNT sponges.

The parameter $t>1$ is due to an increase of the number of contacts between adjacent CNTs and CNT flakes during the compression. To demonstrate this behavior we added additional non-conducting component - cotton wool - to reduce a number of electrical contacts between adjacent flakes within the SWCNT sponge. We found that after the addition of the cotton wool the parameter $t$ decreased up to 1.6. In this way it is possible to tune exponent $t$ to a certain extent.

In narrow density interval, MWCNT sponge recovers its form after removing the compression. In order to show this, we carried out 17 consequent measurements where the sponge was compressed and then released several times. Figure 6 shows the density, reflection, absorptance, and conductivity of the sample at different measurements numbered consequently with $N=1,2 . .17$. One can see that small deformations $(2 \rightarrow 3$, $6 \rightarrow 8,10 \rightarrow 12$, and $13 \rightarrow 15$ ) are elastic and the parameters $\rho, A, R$ and $\sigma$ recovers after removing the compression: they are practically the same at $N=\{2,4\}$, $N=\{5,7,10,13,16\}, N=\{8,11,14\}$, and $N=\{12,15,17\}$ in figure 6. Let us define a compressive strain as a ratio $\left(d-d_{0}\right) / d_{0}$, where $d_{0}$ and $d$ are the thicknesses of the released and compressed samples, respectively; note that $d \propto 1 / \rho$. As follows from figure 6 , the compressive strain at elastic compression $13 \rightarrow 15$ is $42 \%$. Thus, elastic properties of MWCNT sponges may cause a reversible tunability of their electromagnetic parameters at compressive strain $<42 \%$.

Terahertz parameters of MWCNT sponges. Uncompressed, free-standing MWCNT 

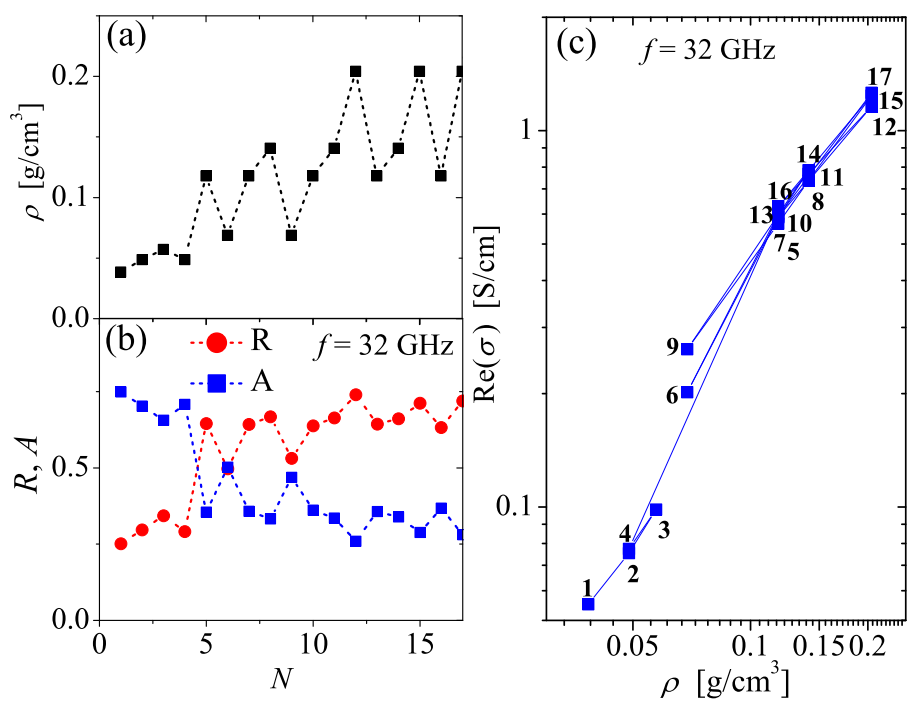

Figure 6. (a) Density $\rho$, (b) absorptance $A$ and reflectance $R$ of the MWCNT sponge at $32 \mathrm{GHz}$ at different measurements carried out in consecutive order indicated with number $N$. The numbers $N=\{2,3,5,7,8,10,11,12,14,15,17\}$ correspond to the sponge under compression whereas $N=\{4,6,9,13,16\}$ indicate the sponge 1 minute after removing the compression. (c) The conductivity versus the density of MWCNT sponge at different measurements indicated with numbers 1 to 17 and mentioned in $(a, b)$.
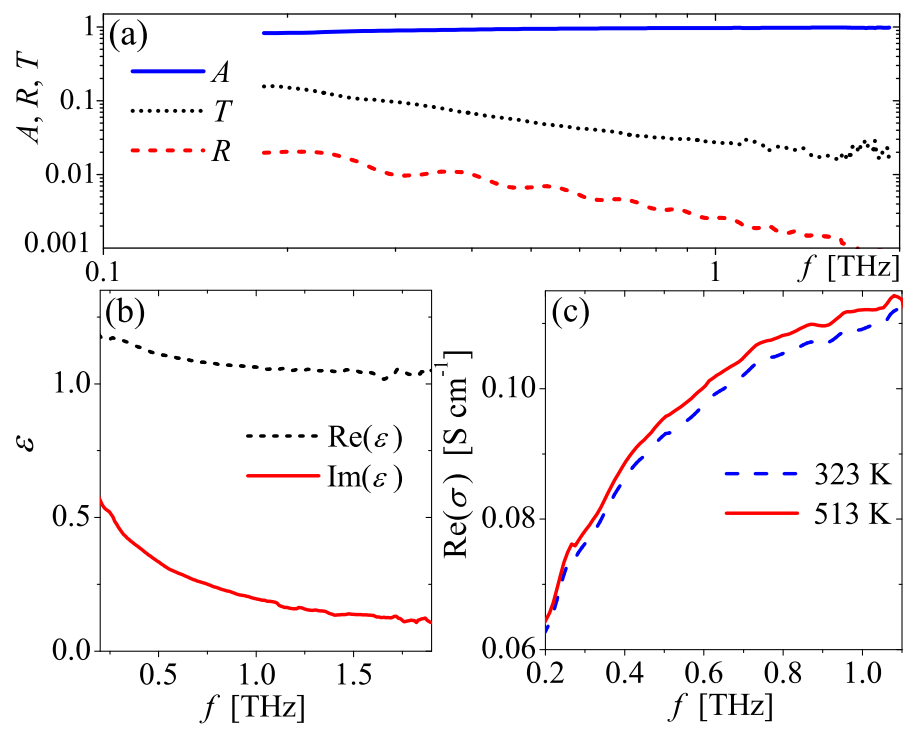

Figure 7. (a) Frequency dependence of (a) the absorptance $A$, transmittance $T$, reflectance $R$ and (b) permittivity of MWCNT sponge at $300 \mathrm{~K}$. (c) Frequency dependence of the conductivity of the MWCNT sponge at $323 \mathrm{~K}$ and $513 \mathrm{~K}$.

sponge with thickness of $0.2 \mathrm{~mm}$ is partly transparent in the terahertz range. Figure 7 (a,b) shows the reflectance $R$, transmittance $T$, absorptance $A$ and the the permittivity of MWCNT sponge at $300 \mathrm{~K}$. 
Let us note small reflectance $(<0.5 \%)$ and high absorptance $(98 \%)$ at frequency of $1 \mathrm{THz}$. Also, the real part of the permittivity is close to unity $1<\operatorname{Re}(\varepsilon)<1.25$ and the imaginary part decreases from 0.55 to 0.12 as frequency increases from 0.2 to $2 \mathrm{THz}$ (see figure $7(\mathrm{~b})$ ).

Figure $7(\mathrm{c})$ shows a temperature variation in the real part of terahertz conductivity of MWCNT sponge while heating from $323 \mathrm{~K}$ to $513 \mathrm{~K}$. The value of $\operatorname{Re}(\sigma)$ increases with frequency demonstrating non-Drude behavior typical for MWCNTs [38, 39]. The conductivity increases slightly with temperature and this can be explained by an increase of a number of conducting channels in thick MWCNTs [40]. Another mechanism - a decrease of electron relaxation time with increasing temperature - causes the opposite temperature dependence of the conductivity [19]. However, this mechanism is not dominant in our sample.

\section{Conclusion}

The microwave measurements of the SWCNT and MWCNT sponges have been carried out in the range 27-36 GHz for different extents of compression. Uncompressed sponges have high absorptance (76\%) and low reflectance $(24 \%)$. The compression leads to an increase in conductivity and, consequently, to a strong variation in the reflectance and absorptance of the samples. Though the qualitative behavior of the microwave response are very similar for both types of sponges, the conductivity of SWCNT sample changes stronger with increasing tube density compared to that for MWCNTs. The density dependence of the microwave conductivity follows the power-law relation $\sigma \propto \rho^{t}$ with $t \in(1.6,1.8)$ and $t \in(1.8,2.2)$ for MWCNT and SWCNT samples, respectively. It is found that the parameter $t$ can be tuned by using additional non-conductive component separating partly CNTs in the sample. The terahertz conductivity of uncompressed MWCNT sponges was shown to increase slightly with temperature that may occur due to an increase of a number of conducting channels in thick MWCNTs.

Thus, we demonstrate a simple and easy way to tune smoothly the electromagnetic response of CNT-sponges in the microwave frequency range through applied mechanical stress to them. Such a systems could be very interesting for different electromagnetic applications, where the tunability is the topical issue, e.g. for passive components of microwave photonics, such as filters, polarizers, collimators and modulators.

\section{Acknowledgments}

The work has partly benefited of funding from the EU H2020 program under the MSCARISE-2014 project 644076 "CoExAN". PK and SM are thankful for support by Tomsk State University Competitiveness Improvement Program. 


\section{References}

[1] Slepyan G Y, Maksimenko S A, Lakhtakia A, Yevtushenko O and Gusakov A V 1999 Phys. Rev. $B \mathbf{6 0} 17136$

[2] Burke P J 2002 IEEE Trans. Nanotechnol. 1129

[3] Jeon T-I, Kim K-J, Kang C, Maeng I H, Son J-H, An K H, Lee J Y and Lee Y H 2004 J. Appl. Phys. 955736

[4] Hanson G 2005 IEEE Trans. Antenn. Propagat. 533426

[5] Slepyan G Y, Shuba M V, Maksimenko S A, Thomsen C and Lakhtakia A. 2010 Phys. Rev. B 81 205423

[6] Shuba M V, Slepyan G Y, Maksimenko S A and Hanson G W 2010 J. Appl. Phys. 108114302

[7] Zhang Q, Haroz E H, Jin Z, Ren L, Wang X, Arvidson R S, Luttge A and Kono J. 2013 Nano Lett. 135991

[8] Shuba M V, Paddubskaya A G, Plyushch A O, Kuzhir P P, Slepyan G Y, Maksimenko S A, Ksenevich V K, Buka P, Seliuta D, Kasalynas I., Macutkevic J, Valusis G, Thomsen C and Lakhtakia A 2012 Phys. Rev. B 85165435

[9] Shuba M V, Melnikov A V, Paddubskaya A G, Kuzhir P P, Maksimenko S A and Thomsen C 2013 Phys. Rev. B 88045436

[10] Shuba M V, Paddubskaya A G, Kuzhir P P, Maksimenko S A, Valusis G, Ivanov M, Banys J, Ksenevich V and Hanson G W 2017 Mater. Res. Express 4075033.

[11] Li H, Yin W Y, Banerjee K and Mao J F 2008 IEEE Trans. Electon. Dev. 551328

[12] Maffucci A, Miano G and Villone F 2009 IEEE Trans. Nanotechnol. 8345

[13] Burke P J, Li S and Yu Z 2006 IEEE Trans. Nanotechnol. 5314

[14] Slepyan G Y, Shuba M V, Maksimenko S A and Lakhtakia A 2006 Phys. Rev. B 7319541

[15] Hartmann R R, Kono J and Portnoi M E 2014 Nanotechnology 25322001

[16] Qin F and Brosseau C 2012 J. Appl. Phys. 111061301

[17] Kuzhir P, Paddubskaya A, Bychanok D, Nemilentsau A, Shuba M, Plusch A, Maksimenko S, Bellucci S, Coderoni L, Micciulla F, Sacco I, Rinaldi G, Macutkevic J, Seliuta D, Valusis G and Banys J 2011 Thin Solid Films 5194114

[18] Bauhofer W and Kovacs J Z 2009 Comp. Sci. Techn. 691486

[19] Karlsen P., Shuba M. V., Beckerleg C, Yuko D I, Kuzhir P P, Maksimenko S A, Ksenevich V, Viet H, Nasibulin A G, Tenne R and Hendry E 2018 J. Phys. D: Appl. Phys. 51014003

[20] Wu J and Kong L 2004 Appl. Phys. Lett. 844956

[21] Hoang A S 2011 Adv. Nat. Sci.: Nanosci. Nanotechnol. 2025007

[22] Gupta T K, Singh B P, Dhakate S R, Singh V N and Mathur R B 2013 J. Mater. Chem. A 19138

[23] Liu Z, Bai G, Huang Y, Li F, Ma Y, Guo T, He X, Lin X, Gao H and Chen Y 2007 J. Phys. Chem. $C 11113696$

[24] Gupta T K, Singh B P, Teotia S, Katyal V, Dhakate S R and Mathur R B 2013 J. Polym. Res. 20169

[25] Zeng Z, Chen M, Jin H, Li W, Xue X., Zhou L, Pei Y, Zhang H and Zhang Z 2016 Carbon 96768

[26] Al-Saleh M H, Saadeh W H, Sundararaj U 2013 Carbon 60146

[27] Bulmer J S, Martens J, Kurzepa L, Gizewski T, Egilmez M, Blamire M G, Yahya N and Koziol K K K 2013 Sci. Rep. 43762

[28] Singh A P, Gupta B K, Mishra M., Govind, Chandra A, Mathur R and Dhawan S 2013 Carbon 5686

[29] Gupta A and Choudhary V 2011 Compos. Sci. Technol. 711563

[30] Stauffer D and Aharony A 1992 Introduction to percolation theory (Taylor Francis, London, UK)

[31] Gui X, Cao A, Wei J, Li H, Jia Y, Li Z, Fan L, Wang K, Zhu H and Wu D 2010 ACS Nano 42320

[32] Camilli L, Pisani C, Passacantando M, Grossi V, Scarselli M, Castrucci P and Crescenzi M D 2013 Appl. Phys. Lett. 102183117.

[33] Scarselli M, Castrucci P, Nicola F De, Cacciotti I, Nanni F, Gatto E, Venanzi M and Crescenzi M 
De 2015 Beilstein J. Nanotechnol. 6792

[34] Gui X, Li H, Wang K, Wei J, Jia Y, Li Z, Fan L, Cao A, Zhu H and Wu D 2011 Acta Mater. 59 4798

[35] Camilli L, Pisani C, Gautron E, Scarselli M, Castrucci P, Orazio F D, Passacantando M, Moscone D and Crescenzi M D 2014 Nanotechnology 25065701.

[36] Chung B K 2007 Prog. Electromagn. Res. 75239

[37] Standard test method for measuring relative complex permittivity and relative magnetic permeability of solid materials at microwave frequencies, astm d5568-08 2009.

[38] Shuba M V, Paddubskaya A G, Kuzhir P P, Maksimenko S A, Flahaut E, Fierro V, Celzard A and Valusis G 2017 J. Phys. D: Appl. Phys. 50 08LT01

[39] Dadrasnia H L E and S. Puthukodan 2014 J. Nanophoton. 88

[40] Maffucci A, Micciulla F, Cataldo A E, Miano G and Bellucci S 2017 IEEE Trans. Compon., Packag., Manuf. Technol. 7485

[41] Paddubskaya A, Valynets N, Kuzhir P, Batrakov K, Maksimenko S, Kotsilkova R, Velichkova H, Petrova I., Biro I, Kertesz K, Mark G I, Horvath Z E and Biro L P 2016 J. Appl. Phys. 119 135102 